\title{
The Many Myths of Erythrocyte Sedimentation Rate and C-Reactive Protein
}

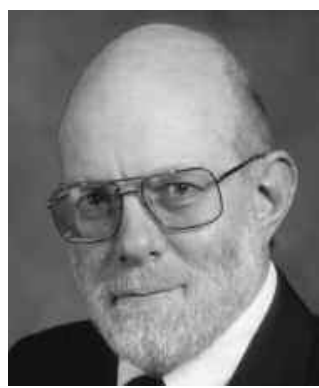

In 2004 Ward analyzed 63 rheumatoid arthritis (RA) clinical trial studies with 90 active disease modifying antirheumatic drug treatment arms and demonstrated that erythrocyte sedimentation rate (ESR) was more sensitive to change than C-reactive protein (CRP) at 12 weeks and 24 weeks of treatment, with mean effect size differences of 0.09 to 0.11 units $^{1}$. It is puzzling that Crowson, et al, authors from the Mayo Clinic and Centocor, address the issues of ESR versus CRP again, and with correlation analyses ${ }^{2}$. If we get their point, it is that it doesn't matter which test is used in a clinical trial as they are both about as effective (or ineffective). This conclusion comes primarily from finding similar levels of correlation between swollen joint count and ESR and $\mathrm{CRP}$, and through analysis of normal values. But correlation analysis, unfortunately, cannot address sensitivity to change, so we cannot assume that the tests are equal, particularly in view of Ward's report ${ }^{1}$. And there are problems with "normal values," as we note below.

The authors then recommend the use of the CRP in clinical trials and clinical practice settings because it is easier and less time-consuming to perform (at the Mayo Clinic). It should be noted that small clinics can perform the simple ESR in their laboratories, but must send CRP determinations to specialty laboratories at additional cost and delay. The central laboratory advantage of the CRP is important to clinical trials, but it is a mythical advantage in clinical practice. There are a number of other myths that are underscored by the current study.

Myth 1. Normal values of ESR and CRP are meaningful in $R A$. Whatever the value of knowing normal values for ESR and CRP in the general population, the values have little meaning in patients with RA who are not representative of the general population ${ }^{3}$. In addition, many people with active RA have normal values of ESR/CRP and many with inactive RA have abnormal values ${ }^{4}$. In the article by Crowson, et al, the normal/abnormal cutoff of ESR and CRP were not determined on paired samples in the same population, virtually guaranteeing non-agreement in normal/abnormal categories for the 2 tests.

Myth 2. There is a rational cutoff for active/inactive $R A$. Rather than distinguishing healthy persons from those with RA, ESR, and CRP are most often used as measures of RA activity. The usual clinical trial activity cutpoints for ESR are $28-30 \mathrm{~mm} / \mathrm{h}$ and for CRP range from 1.0 to 2.0 . However, there is no clear rational cutoff for activity (or for normality) of ESR/CRP in RA ${ }^{4,5}$. Many people with active RA have inactive values of ESR/CRP and many with inactive RA have active values.

Myth 3. Dichotomizing makes sense. Dichotomizing continuous variables loses information and makes for bad groups $^{6}$. This is nowhere more true than in the case of the ESR and CRP. Can it rationally be argued that a patient with an ESR of $27 \mathrm{~mm} / \mathrm{h}$ has inactive RA and one with a value of $28 \mathrm{~mm} / \mathrm{h}$ has active RA?

Myth 4. ESR/CRP are not useful in those with "inactive" $R A$. Data, however, suggest the contrary: that RA is often active in those with inactive RA based on ESR/CRP criteria. We used data on 737 RA patients with 2512 observations (courtesy of the Arthritis and Rheumatology Clinics of Kansas) in which all ESR values were $<28 \mathrm{~mm} / \mathrm{h}$ in a fixed-effect regression analysis. We found that ESR levels were significantly associated with both patient global severity and physician global activity scores. This provides evidence that "non-active" ESR respond to changes in RA activity.

Myth 5. Clinical trial data about ESR/CRP are relevant to clinical practice. Entry into most clinical trials requires elevated ESR or CRP and usually treatment-resistant patients. Such patients are a small and unrepresentative subset of patients compared with community practice. In addition, clinical trials are about the average patient, clinical practice about the individual patient. Learning how each individual patient responds to RA inflammation in terms of ESR/CRP

See Which measure of inflammation to use?, page 1606

Personal non-commercial use only. The Journal of Rheumatology Copyright @ 2009 . All rights reserved. 
enhances the value of the tests. Learning when to ignore a test and when to rely on it lies at the heart of good medical care.

It is, perhaps, time for more research about testing and measurement in clinical practice.

FREDERICK WOLFE, MD,

National Data Bank for Rheumatic Diseases, University of Kansas School of Medicine, 1035 N. Emporia, Suite 288,

Wichita, Kansas 67214, USA

Address correspondence to Dr. Wolfe.

E-mail:fwolfe@arthritis-research.org

\section{REFERENCES}

1. Ward MM. Relative sensitivity to change of the erythrocyte sedimentation rate and serum C-reactive protein concentration in rheumatoid arthritis. J Rheumatol 2004;31:884-95.
2. Crowson CS, Rahman MU, Matteson EL. Which measure of inflammation to use? A comparison of erythrocyte sedimentation rate and $\mathrm{C}$-reactive protein measurements from randomized clinical trials of golimumab in rheumatoid arthritis. J Rheumatol 2009;36:1606-10

3. Ridker PM. High-sensitivity C-reactive protein: potential adjunct for global risk assessment in the primary prevention of cardiovascular disease. Circulation 2001;103:1813-8.

4. Wolfe F, Michaud K. The clinical and research significance of the erythrocyte sedimentation rate. J Rheumatol 1994;21:1227-37.

5. Wolfe F. Comparative usefulness of C-reactive protein and erythrocyte sedimentation rate in patients with rheumatoid arthritis. J Rheumatol 1997;24:1477-85.

6. Royston P, Altman DG, Sauerbrei W. Dichotomizing continuous predictors in multiple regression: a bad idea. StatMed 2006;25:127-41.

J Rheumatol 2009;36:1568-9; doi.10.3899/jrheum.090386 